\title{
Post-B3LYP Functionals Do Not Improve the Description of Magnetic Coupling in Cu(II) Dinuclear Complexes
}

Ramon Costa, Daniel Reta, Ibério de P.R. Moreira, and Francesc Illas

J. Phys. Chem. A, Just Accepted Manuscript • DOI: 10.1021/acs.jpca.7b12663 • Publication Date (Web): 06 Mar 2018

Downloaded from http://pubs.acs.org on March 12, 2018

\section{Just Accepted}

"Just Accepted" manuscripts have been peer-reviewed and accepted for publication. They are posted online prior to technical editing, formatting for publication and author proofing. The American Chemical Society provides "Just Accepted" as a service to the research community to expedite the dissemination of scientific material as soon as possible after acceptance. "Just Accepted" manuscripts appear in full in PDF format accompanied by an HTML abstract. "Just Accepted" manuscripts have been fully peer reviewed, but should not be considered the official version of record. They are citable by the Digital Object Identifier (DOI@). "Just Accepted" is an optional service offered to authors. Therefore, the "Just Accepted" Web site may not include all articles that will be published in the journal. After a manuscript is technically edited and formatted, it will be removed from the "Just Accepted" Web site and published as an ASAP article. Note that technical editing may introduce minor changes to the manuscript text and/or graphics which could affect content, and all legal disclaimers and ethical guidelines that apply to the journal pertain. ACS cannot be held responsible for errors or consequences arising from the use of information contained in these "Just Accepted" manuscripts. 


\title{
Post-B3LYP Functionals Do Not Improve the Description of Magnetic Coupling in $\mathrm{Cu}(\mathrm{II})$ Dinuclear Complexes
}

\author{
Ramon Costa, ${ }^{1,2}$ Daniel Reta, ${ }^{3}$ Ibério de P. R. Moreira, ${ }^{1,4}$ and Francesc Illas*,1,4 \\ ${ }^{1}$ Institut de Química Teòrica i Computacional (IQTCUB), \\ Universitat de Barcelona, C/ Martí i Franquès 1, E-08028 Barcelona, Spain. \\ ${ }^{2}$ Departament de Química Inorgànica i Orgànica, Universitat de Barcelona, \\ C/ Martí i Franquès 1, E-08028 Barcelona, Spain. \\ ${ }^{3}$ School of Chemistry, The University of Manchester, \\ Oxford Road, Manchester, M13 9PL, United Kingdom \\ ${ }^{4}$ Departament de Ciència dels Materials i Química Física, Universitat de \\ Barcelona, C/ Martí i Franquès 1, E-08028 Barcelona, Spain.
}

\begin{abstract}
The accuracy of post-B3LYP functionals is analyzed using an open shell database of $\mathrm{Cu}$ (II) dinuclear complexes with well defined experimental values of the magnetic coupling constants. This database provide a sound open shell training set to be used to improve the fitting schemes in defining new functionals or when reparametrizing the existing ones. For a large set of representative hybrid exchangecorrelation functionals, it is shown that the overall description of moderate to strong antiferromagnetic interactions is significantly more accurate than the description of ferromagnetic or weakly antiferromagnetic interactions. In the case of global hybrids, the most reliable ones have $25-40 \%$ Fock exchange with SOGGA and PBE0 being the most reliable and M06 the exception. For range corrected hybrids, the long range corrected CAM-B3LYP and $\omega$ B97XD provide acceptable results and M11 is comparable but more erratic. It is concluded that the reliability of the calculated values is system and range dependent and this fact introduces a serious warning on the blind use of a single functional to predict magnetic coupling constants. Hence, in order to extract acceptable magnetostructural correlations, a "standardization" of the method to be used is advised to choose the optimal functional.

*Corresponding author: francesc.illas@ub.edu




\section{Introduction.}

One of the goals of quantum chemistry is solving the time-independent Schrödinger equation for $n$ electrons moving in an electric field generated by $N$ (fixed) nuclei. ${ }^{1,2}$ The solution provides the energy and the wave functions of the quantum mechanical states of this system for different nuclear configurations and hence provides a general scheme to obtain equilibrium geometries and properties of the ground state, as well as of excited states. In practice, one focuses on obtaining approximate wave functions $\Psi_{i}\left(\mathbf{r}_{1} s_{1}, \ldots, \mathbf{r}_{\mathrm{n}} s_{\mathrm{n}}\right)$ that explicitly depend on the $3 n$ spatial coordinates ( $4 n$ including spin) of all electrons in the system. From the wave functions one can derive an explicit form of the energy functional in terms of the one- and twoelectron density matrices respectively leading to $E\left[\gamma_{1}\left(\mathbf{r}_{1} ; \mathbf{r}_{1}{ }^{\prime}\right), \gamma_{2}\left(\mathbf{r}_{1}, \mathbf{r}_{2} ; \mathbf{r}_{1}{ }^{\prime}, \mathbf{r}_{2}{ }^{\prime}\right)\right] .12^{3}$ However, the exact solution becomes unattainable except in a finite basis- leading to the well known full configuration interaction expansion- and for a very limited number of electrons only. Therefore, simplified wave functions are normally used as in the case of the Hartree-Fock method, a mean field approximation neglecting instantaneous electron-electron interactions usually referred to as electron correlation effects. Going beyond this approximation becomes soon intractable except for small gas phase molecules thus limiting its application for general purposes.

Density functional theory (DFT) provides an alternative approach to describe the energy $E$ of the electronic ground state of the system of $n$ electrons and $N$ nuclei in terms solely of the electronic density distribution $\rho(\mathbf{r})$ that explicitly depends on 3 coordinates: the trace of the $\gamma_{1}\left(\mathbf{r}_{1} ; \mathbf{r}_{1}^{\prime}\right)$ one electron density matrix. This theory is based on the Hohenberg and Kohn theorems. ${ }^{4}$ The first theorem states that for a system of $n$ electrons and $N$ nuclei in a non-degenerate ground state, the ground state density determines the external potential $\mathrm{V}(\mathbf{r})$ uniquely (except perhaps to an additive constant), and since it also determines $n$ by integration, it determines the full Hamiltonian and (through a big jump in an unknown direction other than solving the Schrödinger equation) all the properties of the system. An important corollary is that the energy of the ground state, $E$, can be obtained from the exact electron density by means of a universal functional $E[\rho(\mathbf{r})]$ of the electron density $\rho(\mathbf{r})$ only. The second theorem states that for any density other than the exact one, the energy obtained from this exact functional is higher than the one corresponding to the exact density which opens a way for practical applications. 
The theorems do not provide the form of this universal, exact, yet unknown, functional and a large theoretical effort has been devoted to justify some of its properties and variational behavior since its introduction in 1964. Soon after, Kohn and Sham $^{5}$ introduced a reference non interacting system to provide a formal structure of the terms in the $E[\rho(\mathbf{r})]$ functional. Using a single Slater determinant to describe $\rho(\mathbf{r})$, the variational procedure leads to a set of one electron equations with the same structure to the Hartree-Fock ones in which the still unknown universal exchangecorrelation energy functional, $E_{x c}[\rho(\mathbf{r})]$, includes all the complicated electron-electron correlation effects as well as the missing kinetic energy terms. ${ }^{6}$

During the last two decades, density functional theory (DFT) has become a fundamental tool for modern quantum-chemical modeling of molecules and materials in many different areas of physics, chemistry and biochemistry. ${ }^{7}$ Large efforts have been developed to produce many different approximations to the unknown $E_{x c}[\rho(\mathbf{r})]$ functional in order to obtain accurate energies and electron densities of molecular and solid state systems and, hence, to attain a useful and reliable description of their physical and chemical properties. To this end, several benchmarks, including thermochemistry and energy barriers for chemical reactions, have been selected to optimize the functional forms of the exchange-correlation potentials in the Kohn-Sham approximation.5 These benchmarks provide experimentally well-defined values which are taken as reference to test functional forms derived from different strategies.

Despite the improvement of the accuracy in describing formation energies of atoms and molecules, bonding energies, molecular and crystal structures, as well as other properties, Perdew and collaborators ${ }^{8}$ pointed out recently that the increasing accuracy attained by post-B3LYP functionals in describing the energy of an electronic system does not necessarily imply a better description of the electron density of the system. For atomic systems, these authors show that even if overparameterized exchange-correlation functionals proposed in the past few years show improved accuracy for the calculated energy, the corresponding densities do not get closer to the exact ones. 8 This tendency is more evident for the functionals proposed since the early 2000 s and the authors associate this drawback to the extensive use of unconstrained functionals that rely on the flexibility of empirical fitting by ignoring the known physical constraints to construct the energy functional. Nevertheless, this view is not universally accepted and subject of some controversy. ${ }^{9-11}$ 
An important remark regarding most of the recently proposed density functionals is that these focus essentially in thermochemistry and pay little attention to other properties that can be experimentally measured and directly determined by the low energy spectrum of the system. This is precisely the case of magnetic coupling $(J)$ in diradicals and some inorganic dinuclear complexes, determined by the energy difference between electronic states with different total spin. Following previous work $^{12,13}$ where a series of functionals were tested against a well defined database of open shell systems, we extend our study to analyze the reliability of functionals derived after the very successful and broadly used B3LYP one. In the following we will refer to these functionals as post-B3LYP and will focus on the magnitude of the magnetic coupling constants of a family of dinuclear $\mathrm{Cu}$ (II) complexes with a variety of bridging ligands ranging from strong antiferromanetic to strong ferromagnetic coupling constants. This allows us to establish an "open shell training set" for the testing and calibration newly designed density functionals. The close connection between the calculated $J$ values and the degree of electron delocalization provides additional information to assess the overall performance of a given functional. In this sense, we will advise that a more accurate description of $J$ values along this open shell training set is an indication of an improved description of the spin density distribution as well. We argue that the relevance of this open shell training set goes beyond the particular case of $\mathrm{Cu}$ (II) complexes and should be included in the benchmarks used to analyze the performance of new functionals.

This paper is organized as follows: in Sec. 2 we provide an account of the different improvement paths in developing the DFT functionals up to now. Next we describe in Sec. 3 the molecular compounds with well-defined crystal structure and experimentally known magnetic coupling to establish an open shell training set. In Sec. 4 we describe the computational details and the scheme used to obtain the suitable energy difference and in Sec. 5 we describe the $J$ values predicted by the different functionals in order to analyze their reliability in describing this relevant molecular property. Finally, in Sec. 6 we summarize our remarks and conclusions.

\section{2.- The open shell database of $\mathrm{Cu}$ (II) dinuclear compounds}

In this section we describe an enlarged set of molecular $\mathrm{Cu}(\mathrm{II})$ magnetic systems introduced in previous works ${ }^{12,13}$ as a reference open shell training set to 
systematically investigate the performance of the post-B3LYP exchange-correlation functionals to predict magnetic coupling constants.

The $\mathrm{Cu}$ (II) dinuclear complexes have been selected in order to minimize ZFS effects, the availability of well characterized molecular crystal structures with a moderate number of atoms, and the diversity of bridging ligands and a wide range of experimentally determined magnetic coupling constants. In order to avoid mixing structural and electronic effects, the crystallographic structures for which the magnetic parameters have been measured have been used without further optimization, except for a few cases where some hydrogen atoms belonging to terminal ligands (not affecting the bridging ones) were not defined: in these cases partial optimizations using B3LYP functional on the triplet states have been used to locate them in approximate positions. To facilitate the calculations of compounds containing ferrocenecarboxylato bridges, these ligands have been replaced by formiato groups, as the magnetic interaction is exclusively mediated by the carboxylate group.

The selected list of dinuclear $\mathrm{Cu}(\mathrm{II})$ complexes covers a broad range of exchange coupling constant $J$ values, ranging from strong antiferromagnetic coupling (singlet-triplet gap of $-400 \mathrm{~cm}^{-1}$ ) to strong ferromagnetic (up to $230 \mathrm{~cm}^{-1}$ gap). They contain a wide diversity of bridging ligands (the ones responsible of the magnetic exchange) including water, hydroxo, O- and $\mathrm{O}, \mathrm{O}$-carboxylates, oxalate derivatives, and 1,1-azido. In order to identify the selected compounds the standard abbreviations used as REFCODEs in the Cambridge Structural Database System ${ }^{14}$ have been used. The details on the molecular structure and properties of some of BISDOW, ${ }^{15}$ CUAQAC02, ${ }^{16}$ CAVXUS, $^{17}$ XAMBUI, ${ }^{18}$ and YAFZOU $^{19}$ have been discussed previously as a reference open shell database by some of us. Here we provide a short description of the main features of the additional complexes considered in this work, namely QAQVIP, PATFOG, JEJCIK and LIZVOF.

QAQVIP $^{20}$ consists of well separated dinuclear cations with two pentacoordinated $\mathrm{Cu}(\mathrm{II})$ atoms each having a chelating bidentate 2,2'-bipyridine terminal ligand and sharing three bridging ligands (three benzoates, two of them acting as bidentate and one as $O, O$-monodentate). One of the $\mathrm{Cu}(\mathrm{II})$ ions is in a mainly square-pyramidal environment where the monodentate bridging benzoate lies in the apical position. The other metal has a trigonal-bipyramidal pentacoordination where the monodentate bridging benzoate lies in one of the equatorial positions. In this way, it is expected that the main interaction between the magnetic orbitals occurs through 
the two bidentate $O, O^{\prime}$-carboxylato bridges. The monodentate bridge does not participate in the superexchange interactions thus avoiding countercomplementary effects through this ligand, so only the antiferromagnetic pathways through both bidentate carboxylates remain active.

The PATFOG cation ${ }^{21}$ consists of a trimetallic complex with a dinuclear copper(II) core where the $\mathrm{Cu}(\mathrm{II})$ ions are bridged by one hydroxo and one $\mu-O, O^{\prime}$ ferrocenecarboxylate anions. Each copper atom is also bound to one terminal bidentate $N, N, N$ ', $N$ '-tetramethylethylenediamine (tmen, Me4en) ligand in such a way that the $\left[\mathrm{CuN}_{2} \mathrm{O}_{3}\right]$ chromophores define the basal planes of a square-pyramidal coordination. The apical positions are occupied by different oxygen atoms of one of the perchlorate anions, which in fact acts as a third $O, O^{\prime}$ bridge with $\mathrm{Cu}-\mathrm{O}$ distances longer than 2.4 $\AA$, so indicating a $4+1$ coordination. Another relevant structural feature of this dimeric cation is the existence of a hydrogen bond between the bridging hydroxo group and one oxygen atom of the other perchlorate anion. The ferrocenyl moiety seems that does not affect the magnetic properties of the system, which exchange is exclusively mediated by its carboxylate group, so to facilitate our calculations it has been replaced by a formiato group.

JEJCIK contains a dinuclear cation ${ }^{22}$ consisting of a triply bridged pair of fivecoordinate copper atoms. Both are in square-planar environments sharing one basal edge and the apical positions. A water molecule acts as the apical bridge whereas a mu-hydroxo and a $\mu-O, O^{\prime}$-acetato are the basal bridging ligands in such a way that the acetato end of the shared edge acts also as a separator. As expected, the apical $\mathrm{Cu}-\mathrm{O}$ distances are noticeably longer than basal bond distances. The $\mathrm{Cu}$ atoms are only 0.092 and $0.137 \AA$ out of the basal planes, which form a 61.9 dihedral angle. The $\mathrm{Cu}$ $\mathrm{O}(\mathrm{H})-\mathrm{Cu}$ bridging angle is $103.8^{\circ}$, a relatively small value that may be contributing to the observed ferromagnetism. The cations form infinite chains as a result of $\mathrm{H}$ bonding interactions with one of the perchlorate anions connecting the $\mathrm{OH}$ - hydrogen atom of one cation and one hydrogen atom of the apical water molecule of an adjacent cation. The weak intermolecular antiferromagnetic interactions observed in the measures at low temperature ${ }^{22}$ are attributed to these contacts.

LIZVOF $^{23}$ consists of well-isolated centrosymmetric dinuclear units in which the copper(II) atoms are bridged by two azido ions in end-on conformation. The $\mathrm{Cu}$ (II) ions present 4 (square-planar) $+1+1$ coordination, where the nitrogen donors of the 4ethylpyridine terminal ligands and the bridging nitrogen atoms of each azido anion 
form the nearly planar $\left[\mathrm{N}_{2} \mathrm{Cu}\left(\mu_{1,1}-\mathrm{N}_{3}\right) 2 \mathrm{CuN}_{2}\right]^{2+}$ core of the complex. Two $\mu-O, O^{\prime}-$ nitrate ions are over and under the above mentioned plane, acting also as weakly bridging ligands and completing the $4+1+1$ coordination around the copper(II) atoms. The four atoms of the $\left[\mathrm{Cu}_{2}(\mathrm{~N} \text {-azido })_{2}\right]$ central square ring of the core are in a plane with $\mathrm{Cu}-\mathrm{N}-\mathrm{Cu}$ angles of $98.3^{\circ}$ and the azido groups form an angle of $31.3(2)^{\circ}$ with it. The intradimer $\mathrm{Cu}-\mathrm{Cu}$ distance is $3.024 \AA$.

References and experimental values of the systems considered in this open shell training set are summarized in Table 1 whereas a schematic representation of the atomic structures of the moieties studied in the present work are displayed in Figure 1. Note that counterions are explicitly included in the calculations when the distance to the metal atom is smaller than $2.5 \AA$. More distant bulky counterions have negligible polarization effect on the central dimeric magnetic unit and are neglected.

\section{Computational details.}

Selected post-B3LYP DFT methods have been used to obtain the appropriate energy values to be used in the corresponding mapping described below. In all cases, the calculations were carried out within the spin-polarized (unrestricted) formalism based on a single determinant and both high-spin and broken symmetry (BS) solutions were considered.

A number of representative hybrid exchange-correlation potentials have been used to explore the reliability of post-B3LYP functionals in describing the magnitude of the magnetic coupling constants of the family of $\mathrm{Cu}$ (II) dinuclear compounds described above. In addition to the standard B3LYP ${ }^{24,25}(20 \% \mathrm{HF}), \mathrm{PBE}^{26,27}$ (25\% $\mathrm{HF})$ and BHandHLYP ${ }^{28}(50 \% \mathrm{HF})$ global hybrid functionals, we used a series of hybrid functionals developed during the last fifteen years in order to analyze the performance of some additional ones and to explore the effect of range separation of the Fock exchange in the functionals that have been accepted to have a large impact in electron localization. However, it is has also been suggested that the remaining part of the exchange-correlation functional also plays a significant role in this aspect (see for instance the performance of M06-L in Ref. 12) and here we will also explore this effect including several functionals developed by the Truhlar's group. To this end we have selected the following DFT functionals:

a) Full range hybrid functionals or global hybrids: TPSSh $(10.0 \% \mathrm{HF})$ proposed by Tao et al. ${ }^{29,30}$; X3LYP $(21.8 \% \mathrm{HF})$ proposed by $\mathrm{Xu}$ and 
Goddard, ${ }^{31}$ APFD (22.95 \% HF) of Austin et al. ${ }^{32}$; the M06 (27.0\% HF), M06$2 \mathrm{X}(54.0 \% \mathrm{FE})^{33}$ and M06-HF $(100.0 \% \mathrm{HF})^{34,35}$ family of meta-GGA hybrid functionals proposed by Zhao and Truhlar as well as their local version M06-L $(0.0 \% \mathrm{HF})^{36}$ for completion since it is one of the most reliable local functional available; and the SOGGA11X (40.15\% HF) proposed by Peverati and Truhlar. $^{37}$

b) Range separation corrected hybrid functionals: (SR \% HF - LR \% HF)

b.1) Short range corrected: HSE06 (25.0\% - 0 \%) proposed by Heyd, Scuseria and Hernzerhof ${ }^{38}$ and the MN12SX (25.0\% - $\left.0 \%\right)$ and N12SX (25.0\% - $\left.0 \%\right)$ proposed by Peverati and Truhlar. ${ }^{39}$

b.2) Long range corrected: $\omega \mathrm{B} 97 \mathrm{XD}(0 \%-100.0 \%)$ of Chai and HeadGordon, ${ }^{40}$ CAM-B3LYP (0 \% - $\left.100.0 \%\right)$ by Yanai, Tew, and Handy, ${ }^{41}$ LC$\omega \mathrm{PBE}(0 \%-100.0 \%)$ implemented by Vydrov et al. ${ }^{42,43}$

b.3) the short and long range corrected functional M11 (42.8\% - $100 \%)$ by Peverati and Truhlar. ${ }^{44}$

For each one of the different systems described above, we have computed the magnetic coupling constant with the use of both the broken symmetry and the triplet solutions in the unrestricted (spin-polarized) formalism of a wide variety of state of the art exchange-correlation DFT hybrid functionals. Clearly, in this type of formalism, the spin symmetry is not guaranteed, ${ }^{45}$ although approximate triplet $(T)$ states have been obtained from the unrestricted Kohn-Sham formalism using a single Slater determinant with two unpaired electrons (i.e., $S_{z}=1$ ), whereas to estimate the energy of the open shell singlet state we have relied on the broken-symmetry $(B S)$ approach imposing $S_{z}=0$. In this way, the singlet-triplet gap energy has been obtained on the basis of the Heisenberg spin model Hamiltonian

$$
H=-J \mathbf{S}_{1} \cdot \mathbf{S}_{2}
$$

and using the appropriate mapping based in the expectation value of the Heisenberg Hamiltonian derived from the single determinant approximation for the $T$ and $B S$ states leads to the approximate relation:

$$
J=2[E(B S)-E(T)]
$$


where $E(B S)$ is the energy of the broken-symmetry state and $E(T)$ is the energy of the spin unrestricted approximation to the triplet state.

All DFT calculations were carried out using suitable Gaussian Type Orbitals (GTO) basis sets. For non-metal atoms, the all-electron standard 6-31G(d) basis set has been selected. For the $\mathrm{Cu}$ atoms, we used all-electron rather large standard basis set GTO 6-3111+G extended with an f-function (exponent $(f)=0.528)$. The basis sets chosen were the same as in previous works. ${ }^{46,47}$ Clearly, relativistic effects are not accounted for and one may wonder which effect these may have on the calculated magnetic coupling constants. This issue has been addressed in previous work for embedded cluster models of superconducting cuprate parent compounds where the magnetic coupling constant is defined in a similar way as the energy difference between singlet and triplet states. ${ }^{48}$ Using different types of wave function based methods it was found that the average effect of the basis set, of the description of the $\mathrm{Cu}$ core electrons (all electron versus relativistic effective core potentials) and of the electronic structure methods (CASPT2 versus DDCI) was roughly 5\%. In any case, the influence of relativistic effects will be identical for all the density functionals considered and, at most, calculated results would have to be equally corrected by a small systematic amount.

All calculations were performed on the crystallographic structures of the isolated bimetallic complexes (cationic or neutral) in vacuo. This choice prevents mixing structural and electronic effects and avoids introducing inaccuracies in the magnetic coupling constants arising from errors in the structure optimization. The full set of DFT based calculations have been carried out using the Gaussian09 suite of programs. $^{49}$

\section{Results and discussion.}

The whole set of calculated $J$ values for the systems studied in this work are summarized in Table 2. Compounds are listed left to right ordered from most antiferromagnetic to most ferromagnetic, and up to down in two different sets to separate global from range separated hybrids. To facilitate the analysis, experimental values are presented in a row between results for the two types of functionals. In each group, the functionals are ordered according to increasing percentage of Fock exchange on the DFT functional. In the lower subset of range separated hybrids, the PBE0 results are included as a reference since most of the functionals are 
modifications of this one. A more graphical picture is provided by Figure 2 where experimental values correspond to the continuous line. This figure clearly show that for each compound a large dispersion is found regarding predictions of the different DFT based methods.

To help to classify the performance of each of the explored methods a statistical analysis has been carried out with results summarized in Tables 3 and 4 reporting results in terms of relative error values of $J_{\text {calc }}$ relative to $J_{\exp }$ (in $\mathrm{cm}^{-1}$ ) using global hybrid DFT functionals and range separated hybrid DFT functionals, respectively. In a similar way, Tables 5 and 6 summarize the corresponding mean absolute error (MAE) values. Finally, $a, b$, and $R$ values for linear regressions between $J_{\text {calc }}$ and $J_{\exp }\left(\mathrm{in} \mathrm{cm}^{-1}\right.$ ) for the full set of compounds and for the FM and AF subsets separately are reported in Table $\mathrm{S} 1$ in the supporting information.

For the cases in the present open shell database, all functionals converge and give ferro- $(\mathrm{F})$ and antiferromagnetic (AF) solutions with ground state compatible with experiment and with the calculated spin density at the metal centers. Nevertheless, the calculated $J$ values show a large dispersion and, in some cases, display large deviations with respect to the experiment as can be seen in Tables 3 and 4 . The effect of the functional is clearly seen in Figure 3 where the deviation from experiment for the whole database is displayed for each DFT based method. This figure shows that the dispersion is huge for functionals not including Fock exchange such as M06-L or just a small amount (10\% in TPSSh) and is progressively reduced with increasing the amount of Fock exchange as in BHandHLYP or M06-2X although not reaching an acceptable accuracy. The importance of the Fock exchange indicates that all explored functionals have difficulties in providing a balanced description of non dynamical and dynamical correlation.

Going in more detail in Tables 3 and 4 one can readily observe that for PATFOG and JEJCIK, with experimentally determined small ferromagnetic couplings, most functionals give relative errors larger than $100 \%$; interestingly M062X and N12SX methods predict acceptable $J$ values for PATFOG but not for JEJCIK. In the case of JEJCIK one can attribute the error to difficulties in the experimental measurement which is based in the use of the the Bleaney-Bowers equation which is only reliable for $\mathrm{T}>50 \mathrm{~K} .{ }^{22}$ Nevertheless, the fact that the same problem is encountered in PATFOG indicates that these functionals have difficulties in describing ferromagnetic interactions. This behavior can be also observed in view of the mean 
absolute error (MAE) for the analyzed functionals reported in Tables 5 and 6. For the full set of complexes under study, $\omega$ B97XD and CAM-B3LYP exhibit the smallest MAE values although with large errors for the ferromagnetic systems whereas N12SX and M06-2X provide a more balanced description of ferro- and antiferromagnetic compounds. Nevertheless, the N12SX performs much better for AF compounds, whereas M06-2X gives best results for the ferromagnetic (FM) ones including a fair description of systems with small $J$ values, either FM or AF. To better analyze this trend, columns 2 and 3 of Tables 5 and 6 consider the relative deviation and MAE values for AF and FM systems separately. In addition, Tables 3 and 4 show that $\omega B 97 X D$ and CAM-B3LYP perform very well for AF coupling constants with rather small relative deviation for BISDOW, CUAQAC02, QAQVIP and larger but acceptable (25\%) for CAVXUS. A closer look shows for CAVXUS this corresponds to absolute errors of less than $5 \mathrm{~cm}^{-1}$. For the FM set, the M06-2X functional gives by far the best mean results even if the relative errors seem important. However, Table 2 makes it clear that the worst cases are XAMBUI with an absolute error of less than 2 $\mathrm{cm}^{-1}$ and JEJCIK with an overestimation of $27 \mathrm{~cm}^{-1}$. This particular compounds shows how severe the problem is in compounds with small $J$ requiring to treat non-dynamical and dynamical correlation on equal foot which is a challenge for DFT based methods.

Finally, we comment on the linear regressions for each individual functional data set (see Table S1) with respect to the experimental values. The analysis of these fits give the best overall correlation coefficients for X3LYP, APFD and PBE0 functionals, although their slopes are strongly deviated from unity, thus indicating different behaviors for FM and AF couplings. Interestingly, a slope closer to 1 corresponds to SOGGA method, which shows a constant deviation of $+45 \mathrm{~cm}^{-1}$. Unfortunately, attempts to take advantage of this correct trend fail for small couplings. The $\omega$ B97XD functional shows a good compromise between a satisfactory prediction of AF coupling constants and a linear correlation with experimental values and concomitant slope close to unity although it tends to overestimate the FM couplings.

A final observation concerns the calculated spin densities in the metal centers. For selected compounds this is reported in Table $\mathrm{S} 2$ of the supporting information. All functionals providing quite accurate numerical $J$ values, especially those in the range separated family, tend to predict similar values of the spin density as well whereas this trend breaks down for functionals performing poorly. This seems to provide an 
indication that it is well possible to obtain a quite good estimate of the magnetic coupling and of the electron density as would be expected for the exact functional.8

\section{Conclusions}

A general observation regarding the complete set of systems studied is that predicted magnetic coupling constants in dinuclear $\mathrm{Cu}(\mathrm{II})$ complexes are system dependent and sensitive to the magnitude and sign of the dominant exchange mechanism.

As a general trend it is observed that MAE errors for range-separated functionals are lower than for global hybrids. The $\omega$ B97XD and CAM-B3LYP, both long range corrected with $100 \%$ Fock exchange, show a very good performance in reproducing the experimental values for AF couplings. On the contrary, M11 and N12SX provide slightly better estimates for FM couplings with noticeable accuracy in the description of AF compounds as well.

Regarding global hybrids, the most reliable results are obtained with those having 25-40\% Fock exchange with SOGGA and PBE0 being the most reliable and M06 the exception. For range corrected hybrids, the long range corrected CAMB3LYP and $\omega \mathrm{B} 97 \mathrm{XD}$ provide acceptable results and M11 is comparable but more erratic. We are confident that this conclusion will hold for other magnetic systems as well.

The present results consistently show that the description of moderate to strong antiferromagnetic interactions is significantly more accurate than the description of ferromagnetic or weakly antiferromagnetic interactions. In general, the description of FM systems appears to be much less reliable than that observed for systems with AF coupling. Moreover, in FM systems a large dispersion of values is obtained for functionals with similar amount of Fock exchange. Clearly, the description of ferromagnetic coupling constants is not satisfactorily reproduced with any of the functionals explored M06-2X (54 \% Fock) and BHandHLYP (50 \% Fock) being those that provide acceptable estimates but with significant erratic deviations especially for moderate to strong positive couplings, and its use is recommend to have a guessing approach only. Taking into account the present results, the most reliable functionals for moderate to strong AF coupled systems are PBE0 and APFD global hybrids and $\omega \mathrm{B} 97 \mathrm{XD}$ and CAM-B3LYP long range corrected hybrids. MN12X short range corrected hybrid provides significant correlation values, especially for FM systems. 
An important dispersion of results is also observed with respect to the experimental values along the set of systems. None of the studied functionals provide an overall balanced description of the singlet triplet energy gap for all the systems. However, from the $J_{\text {calc }}$ vs. $J_{\text {expt }}$ linear regressions, those providing a value of the independent term closest to zero should be the most reliable for magnetostructural correlations. However, it should be emphasized that $a$ and $b$ values of linear regressions strongly depend on the range considered. This is a clear indication that the reliability of the calculated values is system and range dependent and introduces a serious warning on the blind use of a single functional to predict magnetic coupling constants. Hence, it is advised performing a "standardization" of the method to be used against a well defined benchmark set of systems with known values of $J$ with a similar structure and $J$ values. This will allow the possibility of extracting acceptable magnetostructural correlations for a set of similar compounds using the optimal functional.

In conclusion, the present open shell database of $\mathrm{Cu}$ (II) dinuclear complexes provides a sound open shell training set to be used to improve the fitting schemes in defining new functionals or when reparametrizing the existing ones. The present result are likely to be relevant for other properties such as chemical reactivity where intermediates and transition states often exhibit multireference character and well defined experimental data is not always directly available.

\section{Acknowledgments}

This research was supported by the Spanish MINECO, the Agencia Estatal de Investigación (AEI) and Fondo Europeo de Desarrollo Regional (FEDER) through projects CTQ2015-64618-R and CTQ2016-76423-P grants and, in part, by Generalitat de Catalunya (grants 2017SGR13, 2017SGR348 and XRQTC). FI acknowledges additional support from the 2015 ICREA Academia Award for Excellence in University Research. 


\begin{abstract}
ASSOCIATED CONTENT
Supporting Information

The Supporting Information is available free of charge on the ACS Publications website at DOI:

Table S1. $a, b$, and $R$ values for linear regressions between $J_{c a l c}$ and $J_{\exp }\left(\right.$ in $\mathrm{cm}^{-1}$ ) for the full set of compounds and for the FM and AF subsets separately

Table S2. Spin densities on $\mathrm{Cu}(\mathrm{II})$ centres from Mulliken population analysis for selected systems. Positive (negative) values represent $\alpha(\beta)$ spin densities. A single absolute value is provided except in the cases where a significant difference between the two magnetic centers is observed.
\end{abstract}

\title{
AUTHOR INFORMATION
}

\section{Corresponding Author}

* E-mail: francesc.illas@ub.edu

Notes

The authors declare no competing financial interest. 
Table 1.Cambridge Crystallographic Data Base (CCDB) names, experimental J values (in $\mathrm{cm}^{-1}$ ) and reference of the molecular systems studied.

\begin{tabular}{|c|c|c|c|}
\hline Complex & CCDB Name & J & Reference \\
\hline $\mathbf{1}$ & BISDOW & -382.00 & 15 \\
\hline $\mathbf{2}$ & CUAQAC02 & -286.00 & 16 \\
\hline $\mathbf{3}$ & QAQVIP & -39.70 & 20 \\
\hline $\mathbf{4}$ & CAVXUS & -19.00 & 17 \\
\hline $\mathbf{5}$ & XAMBUI & 2.50 & 18 \\
\hline $\mathbf{6}$ & PATFOG & 29.00 & 21 \\
\hline $\mathbf{7}$ & JEJCIK & 38.00 & 22 \\
\hline $\mathbf{8}$ & YAFZOU & 111.00 & 19 \\
\hline $\mathbf{9}$ & LIZVOF & 230.00 & 23 \\
\hline
\end{tabular}


Table 2.Calculated DFT J values (in $\mathrm{cm}^{-1}$ ) of the molecular systems studied using the broken symmetry approach for global and range separated functionals.

\begin{tabular}{|c|c|c|c|c|c|c|c|c|c|c|}
\hline HF $\%$ & Functional & bisdow & cuaqac02 & qaqvip & cavxus & xambui & patfog & jejcik & yafzou & lizvof \\
\hline 0 & M06-L & -1316.0 & -752.0 & -138.7 & -25.0 & 23.0 & 119.7 & 273.6 & 334.0 & 139.3 \\
\hline 10 & TPSSh & -918.9 & -586.8 & -105.3 & -26.9 & 8.8 & 52.4 & 173.4 & 193.4 & 226.9 \\
\hline 20 & B3LYP & -633.9 & -429.4 & -70.9 & -21.2 & 3.9 & 74.7 & 163.4 & 193.6 & 304.2 \\
\hline 21.8 & X3LYP & -578.0 & -398.4 & -63.4 & -19.2 & 3.5 & 74.7 & 157.4 & 186.7 & 322.3 \\
\hline 22.945 & APFD & -546.5 & -378.8 & -58.7 & -18.3 & 3.4 & 74.1 & 151.2 & 179.7 & 345.0 \\
\hline 25 & PBE0 & -492.0 & -346.0 & -51.6 & -16.0 & 3.0 & 71.8 & 143.0 & 170.0 & 358.7 \\
\hline 25 & HSE06 & -506.0 & -354.2 & -54.2 & -16.7 & 3.2 & 71.4 & 142.7 & 169.2 & 360.5 \\
\hline 27 & M06 & -632.0 & -436.0 & -71.8 & -28.0 & 3.0 & 137.9 & 229.5 & 294.0 & 385.8 \\
\hline 40.15 & SOGGA & -284.3 & -220.4 & -26.4 & -9.8 & 1.1 & 58.8 & 108.6 & 128.8 & 348.5 \\
\hline 50 & BHandHLYP & -160.2 & -132.0 & -12.4 & -5.3 & 0.7 & 45.6 & 80.4 & 91.0 & 349.3 \\
\hline 54 & M06-2X & -177.0 & -143.0 & -13.0 & -6.0 & 0.8 & 29.8 & 65.1 & 75.0 & 285.0 \\
\hline 100 & M06-HF & -64.0 & -44.0 & -1.0 & -1.0 & 0.2 & -7.3 & 12.3 & 11.0 & 147.7 \\
\hline & EXP & -382.0 & -286.0 & -39.7 & -19.0 & 2.5 & 29.0 & 38.0 & 111.0 & 230.0 \\
\hline $0-100$ & $\omega$ B97XD & -391.0 & -296.0 & -40.1 & -14.4 & 1.6 & 68.4 & 142.7 & 170.4 & 371.1 \\
\hline $0-100$ & CAM-B3LYP & -392.5 & -291.9 & -39.9 & -13.9 & 1.7 & 68.3 & 136.8 & 161.8 & 390.2 \\
\hline $0-100$ & LC- $\omega$ PBE & -497.9 & -271.3 & -36.3 & -14.1 & 1.5 & 57.8 & 130.9 & 153.7 & 464.9 \\
\hline $42.8-100$ & M11 & -221.1 & -167.3 & -16.8 & -8.6 & 0.3 & 43.7 & 82.5 & 100.9 & 354.5 \\
\hline $25-25$ & PBE0 & -492.0 & -346.0 & -51.6 & -16.0 & 3.0 & 71.8 & 143.0 & 170.0 & 358.7 \\
\hline 25 & HSE06 & -506.0 & -354.2 & -54.2 & -16.7 & 3.2 & 71.4 & 142.7 & 169.2 & 360.5 \\
\hline $25-0$ & N12SX & -462.3 & -324.8 & -48.7 & -17.5 & 1.4 & 36.4 & 111.7 & 132.4 & 446.0 \\
\hline $25-0$ & MN12SX & -497.4 & -365.4 & -47.7 & -15.0 & 1.5 & 51.0 & 146.1 & 183.0 & 279.7 \\
\hline & & & & & & & & & \\
\hline
\end{tabular}


Table 3. Relative deviation (in \%) of calculated DFT $J$ values with respect to the experiment of the selected global hybrid DFT functionals. Color code: green (0-25\%), yellow (25-50\%), green (50-75\%), pink (75-100\%) and red (>100\%). Values in blue indicate overestimation with respect the experimental value with sign.

\begin{tabular}{|c|l|c|c|c|c|c|c|c|c|c|}
\hline HF $\%$ & Functional & BISDOW & CUAQAC02 & QAQVIP & CAVXUS & XAMBUI & PATFOG & JEJCIK & YAFZOU & LIZVOF \\
\hline 0 & M06-L & -245 & -163 & -249 & -32 & 820 & 313 & 620 & 201 \\
\hline 10 & TPSSh & -141 & -105 & -165 & -41 & 253 & 81 & 356 & 74 \\
\hline 20 & B3LYP & -66 & -50 & -79 & -12 & 56 & 158 & 330 & 74 \\
\hline 21,8 & X3LYP & -51 & -39 & -60 & -1 & 40 & 158 & 314 & 68 & 40 \\
\hline 22,95 & APFD & -43 & -32 & -48 & 4 & 34 & 155 & 298 & 62 \\
\hline 25 & PBE0 & -29 & -21 & -30 & 16 & 20 & 148 & 276 & 53 & 56 \\
\hline 40,15 & SOGGA & 26 & 23 & 34 & 48 & -54 & 103 & 186 & 16 & 52 \\
\hline 50 & BHandHLYP & 58 & 54 & 69 & 72 & -72 & 57 & 112 & -18 & 52 \\
\hline 54 & M06-2X & 54 & 50 & 67 & 68 & -68 & 3 & 71 & -32 & 24 \\
\hline 100 & M06-HF & 83 & 85 & 97 & 95 & -92 & -125 & -68 & -90 & -36 \\
\hline
\end{tabular}


Table 4. Relative deviation (in \%) of calculated DFT $J$ values with respect to the experiment of the selected range separated hybrid DFT functionals. The HF \% fraction in the short and long ranges of the exchange functional (S-L \%HF) is indicated for each functional. The PBE0 global hybrid is included for comparison to complete the transition between short range and long range correction schemes. Color code: green (0-25\%), yellow (25-50\%), green (50-75\%), pink (75-100\%) and red (>100 \%). Values in blue indicate overestimation with respect the experimental value with sign.

\begin{tabular}{|c|c|c|c|c|c|c|c|c|c|c|}
\hline S-L HF \% & Functional & BISDOW & CUAQAC02 & QAQVIP & CAVXUS & XAMBUI & PATFOG & JEJCIK & YAFZOU & LIZVOF \\
\hline $0-100$ & $\omega \mathrm{B} 97 \mathrm{XD}$ & -2 & -4 & -1 & 24 & -36 & 136 & 275 & 54 & 61 \\
\hline $0-100$ & CAM-B3LYP & -3 & -2 & -1 & 27 & -30 & 135 & 260 & 46 & 70 \\
\hline $0-100$ & LC- $\omega \mathrm{PBE}$ & -30 & 5 & 9 & 26 & -39 & 99 & 244 & 38 & 102 \\
\hline $42,8-100$ & M11 & 42 & 42 & 58 & 55 & -86 & 51 & 117 & -9 & 54 \\
\hline $25-25$ & PBE0 & -29 & -21 & -30 & 16 & 20 & 148 & 276 & 53 & 56 \\
\hline $25-0$ & HSE06 & -32 & -24 & -37 & 12 & 29 & 146 & 276 & 52 & 57 \\
\hline $25-0$ & N12SX & -21 & -14 & -23 & 8 & -44 & 26 & 194 & 19 & 94 \\
\hline $25-0$ & MN12SX & -30 & -28 & -20 & 21 & -40 & 76 & 285 & 65 & 22 \\
\hline
\end{tabular}


Table 5. Mean absolute error (MAE) values for $J_{\text {calc }}$ with respect to $J_{\text {expt }}$ (in $\mathrm{cm}^{-1}$ ) using global hybrid DFT functionals. Different ranges of $J$ values are explored.

\begin{tabular}{|c|c|c|c|c|c|}
\hline HF $\%$ & Functional & (ALL) & (AF) & (FM) & $\mathbf{- 4 0 <} \mathbf{J 3 ~}^{\mathbf{3 0}} \mathbf{~ m}^{-1}$ \\
\hline 0 & M06-L & 241 & 376 & 132 & 54 \\
\hline 10 & TPSSh & 129 & 228 & 50 & 26 \\
\hline 20 & B3LYP & 84 & 107 & 66 & 20 \\
\hline 21,8 & X3LYP & 74 & 83 & 67 & 18 \\
\hline 22,95 & APFD & 69 & 69 & 69 & 16 \\
\hline 25 & PBE0 & 58 & 46 & 67 & 15 \\
\hline 27 & M06 & 120 & 110 & 128 & 131 \\
\hline 40,15 & SOGGA & 47 & 46 & 48 & 15 \\
\hline 50 & BHandHLYP & 69 & 104 & 40 & 38 \\
\hline 54 & M06-2X & 56 & 97 & 24 & 13 \\
\hline 100 & M06-HF & 96 & 154 & 49 & 15 \\
\hline
\end{tabular}


Table 6. Mean absolute error (MAE) values for $J_{\text {calc }}$ with respect to $J_{\text {expt }}$ (in $\mathrm{cm}^{-1}$ ) using range separated hybrid DFT functionals. Different ranges of $J$ values are explored and the PBE0 global hybrid is included for comparison.

\begin{tabular}{|c|c|c|c|c|c|}
\hline HF \% & Functional & (ALL) & (AF) & (FM) & $\mathbf{- 4 0}<\mathbf{J}<\mathbf{3 0 ~ \mathbf { ~ m } ^ { - 1 }}$ \\
\hline $0-100$ & $\omega B 97 X D$ & 41 & 6 & 69 & 11 \\
\hline $0-100$ & CAM-B3LYP & 41 & 5 & 70 & 11 \\
\hline $0-100$ & LC- $\omega$ PBE & 60 & 35 & 80 & 10 \\
\hline $42,8-100$ & M11 & 57 & 78 & 39 & 13 \\
\hline $25-25$ & PBE0 & 58 & 46 & 67 & 15 \\
\hline $25-0$ & HSE06 & 61 & 52 & 67 & 15 \\
\hline $25-0$ & N12SX & 50 & 32 & 51 & 5 \\
\hline $25-0$ & MN12SX & 51 & 52 & 64 & 9 \\
\hline
\end{tabular}


Figure 1. Molecular and ionic fragments of compounds 1-9 studied in the present work

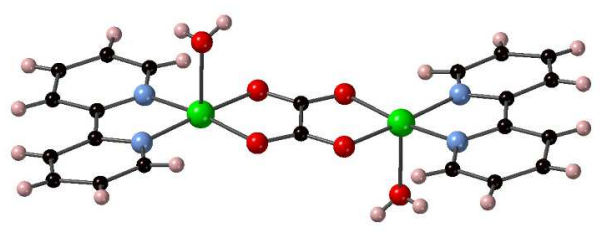

BISDOW

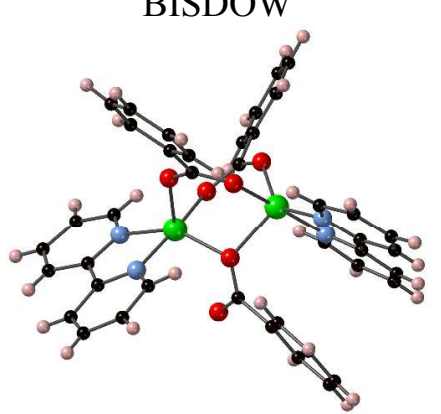

QAQVIP

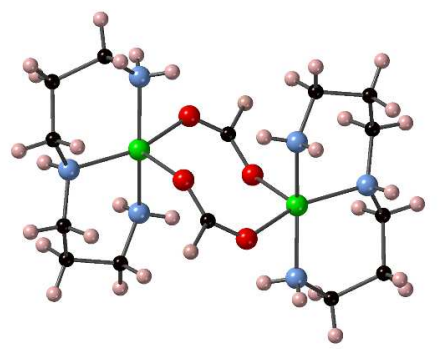

XAMBUI

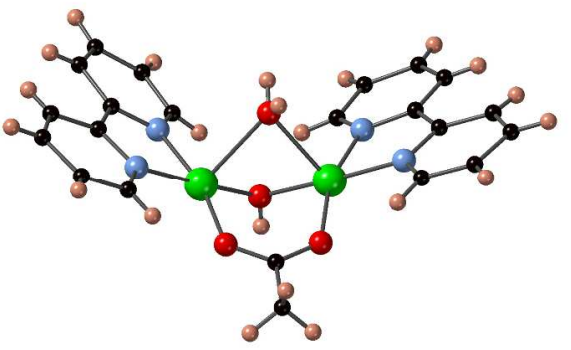

JEJCIK

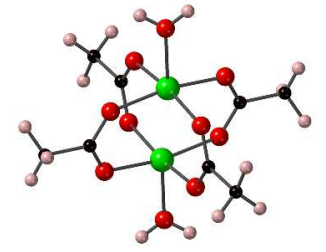

CUAQAC02

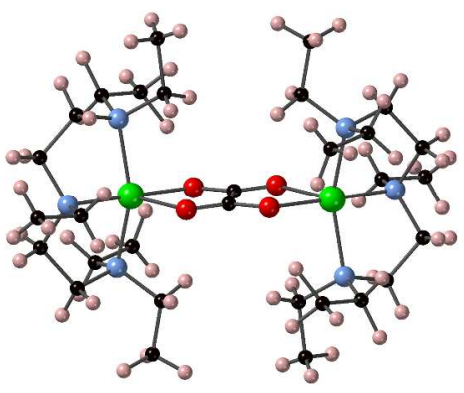

CAVXUS

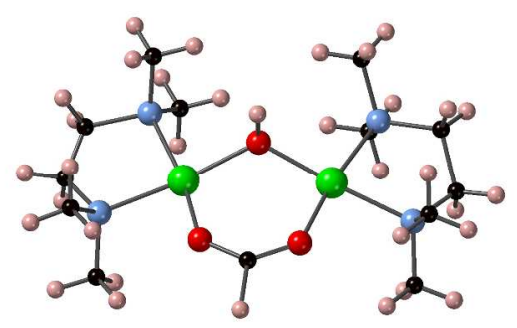

PATFOG

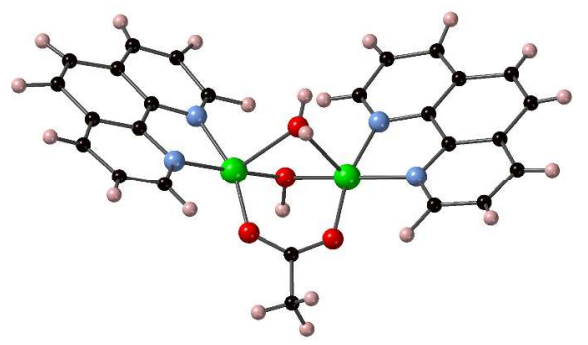

YAFZOU

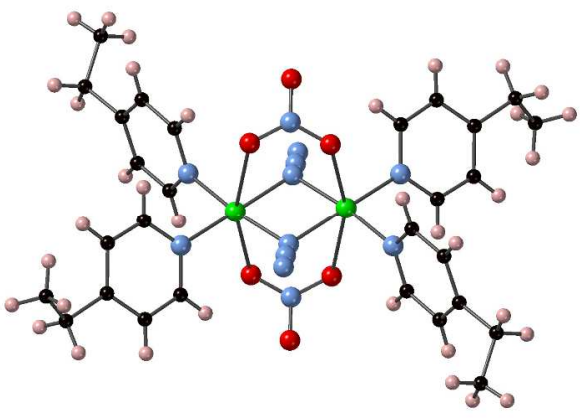

LIZVOF 
Figure 2. Magnetic coupling constant of the compounds studied in the present work as predicted by the different exchange correlation functionals. The continuous line correspond to the experimental values.

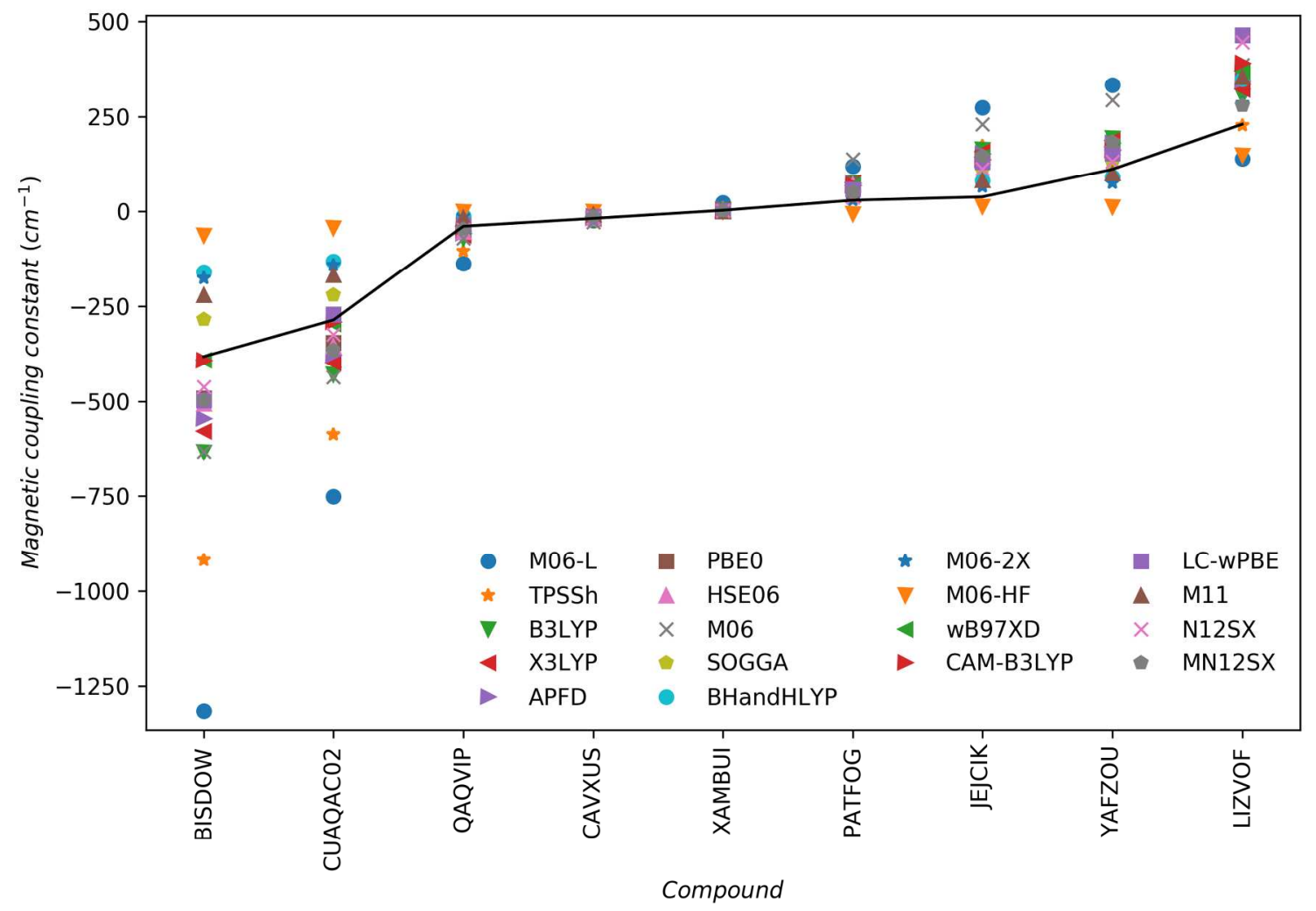


Figure 3. For each of the compounds on the data base, deviation from experiment of values calculated with each different DFT based method.

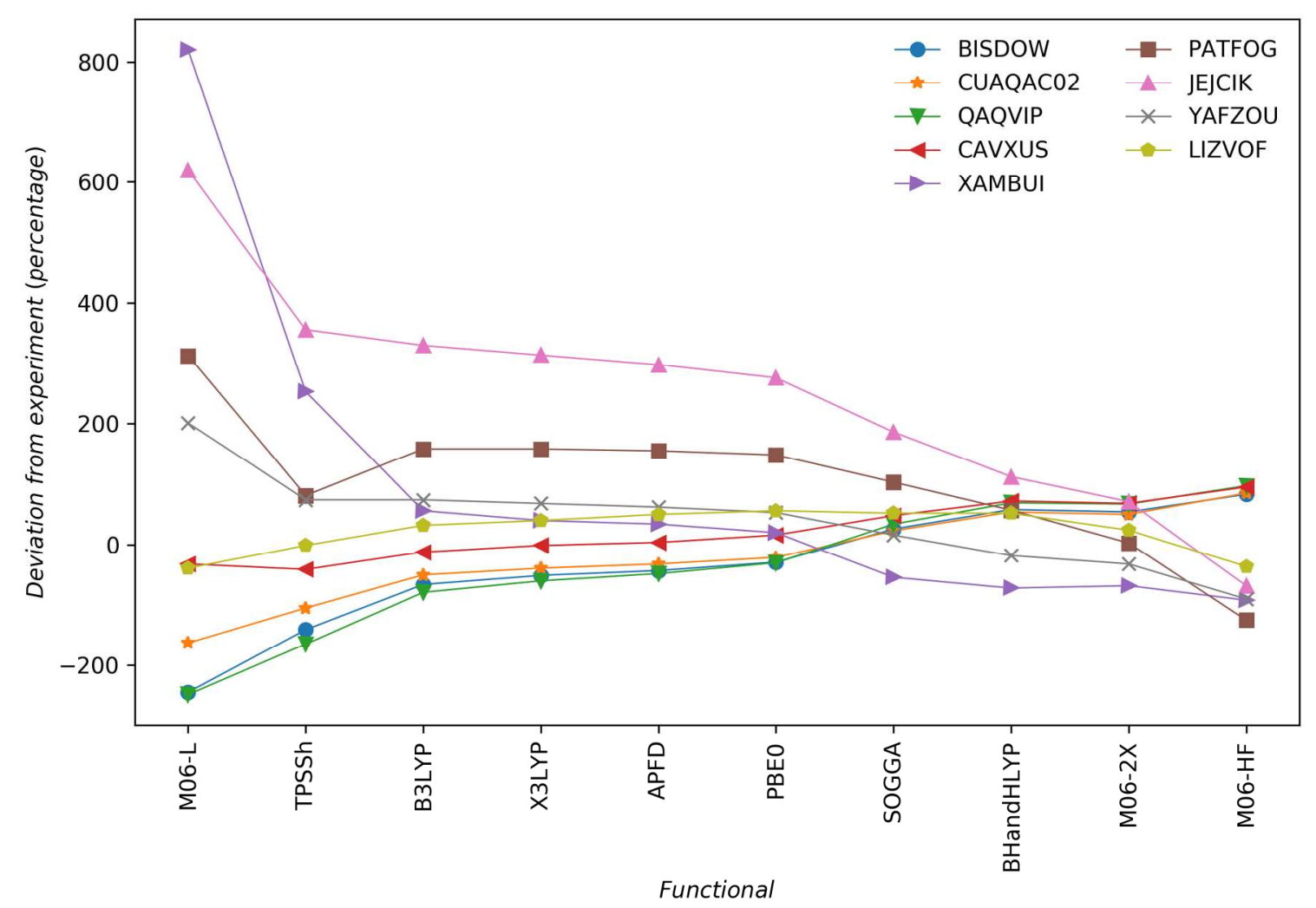




\section{References}

1 Garrod C.; Percus, J.K. Reduction of N-Particle Variational Problem. J. Math. Phys. 1964, 5, 1756.

2 McWeeny, R. Methods of Molecular Quantum Mechanics, 2nd ed. (Academic Press, Oxford, 1992).

3 Caballero, M.; Moreira, I. de P. R.; Bofill, J. M. A Comparison Model between Density Functional and Wave Function Theories by means of the Lowdin Partitioning Technique. J. Chem. Phys. 2013, 138, 174107.

4 Hohenberg, P.; Kohn, W. Inhomogeneous Electron Gas. Phys. Rev. 1964, 136, B864B871.

5 Kohn, W.; Sham, L. J. Self-Consistent Equations Including Exchange and Correlation Effects. Phys. Rev. 1965, 140, A1133-A1138.

6 Parr, R.G.; Yang, W. in Density-Functional Theory of Atoms and Molecules, Oxford University Press, New York, 1989.

7 Jones, R. O. Density Functional Theory: Its Origins, Rise to Prominence, and Future. Rev. Mod. Phys. 2015, 87, 897-923.

8 Medvedev, M. G.; Bushmarinov, I. S.; Sun, J.; Perdew, J. P.; Lyssenko, K. A. Density Functional Theory is Straying from the Path Toward the Exact Functional. Science 2017, $355,49-52$.

9 Wang, Y.; Wang, X. W.; Truhlar, D. G.; He, X. How Well Can the M06 Suite of Functionals Describe the Electron Densities of $\mathrm{Ne}, \mathrm{Ne}^{6+}$, and $\mathrm{Ne}^{8+}$ ?. J. Chem. Theory Comput. 2017, 13, 6068-6077.

${ }^{10}$ Ranasinghe, D. S.; Perera, A.; Bartlett, R. J. A note on the accuracy of KS-DFT densities. J. Chem. Phys. 2017, 147, 204103.

${ }_{11}$ Mezei, P. D.; Csonka, G. I.; Kallay, M. Electron Density Errors and Density-Driven Exchange-Correlation Energy Errors in Approximate Density Functional Calculations. $J$. Chem. Theory Comput. 2017, 13, 4753-4764.

12 Valero, R.; Costa, R.; Moreira, I. de P. R.; Truhlar, D. G.; Illas, F. Performance of the M06 Family of Exchange-Correlation Functionals for Predicting Magnetic Coupling in Organic and Inorganic Molecules. J. Chem. Phys. 2008, 128, 114103. 
13 Rivero, P.; Moreira, I. de P. R.; Illas, F.; Scuseria, G. E. Reliability of Range Separated Hybrids Functionals in Describing Magnetic Coupling in Molecular Systems. J. Chem. Phys. 2008, 129, 184110.

${ }^{14} \mathrm{http}: / /$ www.ccdc.cam.ac.uk/products/csd/. The crystal structure data were downloaded in CIF format from the CCDC database with the help of the ConQuest utility.

15 Castillo, O.; Muga, I;. Luque, A.; Gutierrez-Zorrilla, J.M.; Sertucha, J.; Vitoria, P.; Roman, P. Synthesis, Chemical Characterization, X-Ray Crystal Structure and Magnetic Properties of Oxalato-Bridged Copper(II) Binuclear Complexes with 2,2 '-Bipyridine and Diethylenetriamine as Peripheral Ligands. Polyhedron 1999, 18, 1235-1245.

16 de Meester, P.; Fletcher, S.R.; Skapski, A.C. Refined Crystal-Structure of Tetra-MuAcetato-Bisaquodicopper(II). J. Chem. Soc., Dalton Trans. 1973, 2575-2578.

17 Sletten, J. The Structures of 2 Oxalato-Bridged Cu-Dimers $\left[\mathrm{Cu}_{2}\left(\mathrm{Me}_{4} \mathrm{En}\right)_{2}\left(\mathrm{C}_{2} \mathrm{O}_{4}\right)\left(\mathrm{H}_{2} \mathrm{O}\right)_{2}\right]\left(\mathrm{PF}_{6}\right)_{2} \quad 2 \mathrm{H}_{2} \mathrm{O}$ and $\left[\mathrm{Cu}_{2}\left(\mathrm{Et}_{5} \text { Dien }\right)_{2}\left(\mathrm{C}_{2} \mathrm{O}_{4}\right)\right]\left(\mathrm{PF}_{6}\right)_{2}$. Acta Chem. Scand. A 1983, 37, 569-578.

18 Lopez, C.; Costa, R.; Illas, F.; Molins E.; Espinosa, E. The First Ferromagnetic Copper (II) Complex Containing Ferrocenecarboxylato Bridging Ligands. Inorg. Chem. 2000, $39,4560-4565$.

19 Tokii, T.; Hamamura, N.; Nakashima, M.; Muto, Y. Crystal-Structures and MagneticProperties of Novel Mu-Carboxylato-Mu-Hydroxo-Bridged Binuclear Copper(II) Complexes with 1,10-Phenanthroline. Bull. Chem. Soc. Jpn. 1992, 65, 1214-1219.

${ }^{20}$ Wannarit, N.; Siriwong, K.; Chaichit, N.; Youngme, S.; Costa, R.; Moreira, I. de P.R.; Illas, F. A New Series of Triply-Bridged Dinuclear Cu(II) Compounds: Synthesis, Crystal Structure, Magnetic Properties and Theoretical Study. Inorg. Chem. 2011, 50, 10648-10659.

21 Lopez, C.; Costa, R.; Illas, F.; de Graaf, C.; Turnbull, M.; Landee, C.P.; Espinosa, E.; Mata, I.; Molins E. Magnetostructural Correlations in Binuclear Copper(II) Compounds Bridged, a Ferrocenecarboxylato(-1) and an Hydroxo- or Methoxo- Ligands: Tuning the Sign of Superexchange in Heterobridged Complexes. Dalton Trans. 2005, 2322-2330.

22 Christou, G.; Perlepes, S.P.; Folting, K.; Huffman, J.C.; Webb, R.J.; Hendrickson, D.N. A New Class of Bipyridine-Ligated Metal Carboxylate Complexes - Characterization of 
The Triply-Bridged Ferromagnetically-Coupled Complexes $\left[\mathrm{Cu}_{2}(\mathrm{Oac})_{3}(\mathrm{Bpy})_{2}\right]\left(\mathrm{ClO}_{4}\right)$ and $\left[\mathrm{Cu}_{2}(\mathrm{OH})\left(\mathrm{H}_{2} \mathrm{O}\right)(\mathrm{Oac})(\mathrm{Bpy})_{2}\right]\left(\mathrm{ClO}_{4}\right)_{2}$. Chem. Commun. 1990,746-747.

23 Escuer, A.; Goher, M.A.S.; Mautner, F.A.; Vicente, R. Three New Polynuclear Copper(II) Complexes with the Symmetric $\left[\mathrm{Cu}\left(\mu_{1,1}-\mathrm{N}_{3}\right)_{2} \mathrm{Cu}\right]^{2+}$ Core and Pyridine Derivatives: Syntheses, Structure, and Magnetic Behavior Inorg. Chem. 2000, 39, 21072112.

24 Becke, A. D. Density-Functional Thermochemistry.3. The Role Of Exact Exchange. $J$. Chem. Phys. 1993, 98, 5648-5652.

25 Lee, C.; Yang, W.; Parr, R. G. Development of the Colle-Salvetti Correlation-Energy Formula into a Functional of the Electron-Density. Phys Rev B 1988, 37, 785-789.

26 Adamo, C.; Barone, V. Toward Reliable Density Functional Methods without Adjustable Parameters: The PBE0 Model. J. Chem. Phys., 110 (1999) 6158-6170.

27 Ernzerhof, M.; Scuseria, G. E. Assessment of the Perdew-Burke-Ernzerhof ExchangeCorrelation Functional. J. Chem. Phys., 1999, 110, 5029-5036

28 Becke, A. D. A New Mixing of Hartree-Fock and Local Density-Functional Theories. $J$. Chem. Phys. 1993, 98, 1372-1377.

29 Tao, J. M.; Perdew, J. P.; Staroverov, V. N.; Scuseria, G. E. Climbing the Density Functional Ladder: Nonempirical Meta-Generalized Gradient Approximation Designed for Molecules and Solids. Phys. Rev. Lett. 2003, 91, 146401.

30 Staroverov, V. N.; Scuseria, G. E.; Tao, J. M.; Perdew, J. P. Comparative Assessment of a New Nonempirical Density Functional: Molecules and Hydrogen-bonded Complexes. J. Chem. Phys., 2003, 119, 12129. ibid. 2004, 121, 11507(Erratum).

$31 \mathrm{Xu}, \mathrm{X}$; Goddard III, W. A. The X3LYP Extended Density Functional for Accurate Descriptions of Nonbond Interactions, Spin States, and Thermochemical Properties. Proc. Natl. Acad. Sci. USA 2004, 101, 2673-77.

32 Austin, A.; Petersson, G. A.; Frisch, M. J.; Dobek, F. J.; Scalmani, G.; Throssell, K. A Density Functional with Spherical Atom Dispersion Terms. J. Chem. Theory Comput. 2012, 8, 4989-5007.

33 Zhao, Y.; Truhlar, D. G. The M06 Suite of Density Functionals for Main Group Thermochemistry, Thermochemical Kinetics, Noncovalent Interactions, Excited States, 
and Transition Elements: Two New Functionals and Systematic Testing of Four M06Class Functionals and 12 Other Functionals. Theor. Chem. Acc. 2008, 120, 215-241.

34 Zhao, Y.; Truhlar, D. G. Comparative DFT Study of Van der Waals Complexes: RareGas Dimers, Alkaline-Earth Dimers, Zinc Dimer, and Zinc-Rare-Gas Dimers. J. Phys. Chem. A 2006, 110, 5121-5129;

35 Zhao, Y.; Truhlar, D. G. Density Functional for Spectroscopy: No Long-Range SelfInteraction Error, Good Performance for Rydberg and Charge-Transfer States, and Better Performance on Average than B3LYP for Ground States. J. Phys. Chem. A 2006, $110,13126-13130$.

36 Zhao, Y.; Truhlar, D. G. A New Local Density Functional for Main-Group Thermochemistry, Transition Metal Bonding, Thermochemical Kinetics, and Noncovalent Interactions. J. Chem. Phys. 2006, 125, 194101.

37 Peverati, R.; Truhlar, D. G. A Global Hybrid Generalized Gradient Approximation to the Exchange-Correlation Functional that Satisfies the Second-Order Density-Gradient Constraint and Has Broad Applicability in Chemistry. J. Chem. Phys. 2011, 135, 191102.

38 Heyd, J.; Scuseria, G. E.; Ernzernhof, M. Hybrid Functionals Based on a Screened Coulomb Potential. J. Chem. Phys. 2003, 118, 8207-8215; ibid. 2006, 124, 219906 (E).

39 Peverati, R.; Truhlar, D. G. Screened-Exchange Density Functionals with Broad Accuracy for Chemistry and Solid-State Physics. Phys. Chem. Chem. Phys. 2012, 14, 16187-16191.

40 Chai, J.-D.; Head-Gordon, M. Long-Range Corrected Hybrid Density Functionals with Damped Atom-Atom Dispersion Corrections. Phys. Chem. Chem. Phys. 2008, 10, 66156620

41 Yanai, T.; Tew, D.; Handy, N. A New Hybrid Exchange-Correlation Functional Using the Coulomb-Attenuating Method (CAM-B3LYP). Chem. Phys. Lett. 2004, 393, 51-57.

42 Vydrov O. A.; Scuseria, G. E. Assessment of a Long-Range Corrected Hybrid Functional. J. Chem. Phys. 2006, 125, 234109.

43 Vydrov, O. A.; Heyd, J.; Krukau, A. V.; Scuseria, G. E. Importance of Short-Range versus Long-Range Hartree-Fock Exchange for the Performance of Hybrid Density Functionals. J. Chem. Phys. 2006, 125, 074106. 
44 Peverati, R.; Truhlar, D. G. Improving the Accuracy of Hybrid Meta-GGA Density Functionals by Range Separation. J. Phys. Chem. Lett. 2011,2, 2810-2817.

45 Moreira, I. de P. R.; Costa, R.; Filatov, M.; Illas, F. Restricted Ensemble-Referenced Kohn Sham versus Broken Symmetry Approaches in Density Functional Theory: Magnetic Coupling in $\mathrm{Cu}$ Binuclear Complexes. J. Chem. Theory and Comput.2007, 3, 764-774.

46 Costa, R.; Moreira, I. de P.R.;Youngme, S.; Siriwong, K.; Wannarit, N.; Illas, F. Towards the Design of Ferromagnetic Molecular Complexes: Magnetostructural Correlations in Ferromagnetic Triply-Bridged Dinuclear Cu(II) Compounds Containing Carboxylato and Hydroxo Bridges. Inorg. Chem. 2010, 49, 285-294.

47 Wannarit, N.; Pakawatchai, Ch.; Mutikainen, I.; Costa, R.; Moreira, I. de P. R.; Youngme, S.; Illas, F. Hetero Triply-Bridged Dinuclear Copper(II) Compounds with Ferromagnetic Coupling: A Challenge for Current Density Functionals. Phys. Chem. Chem. Phys. 2013, 15, 1966-1975.

48 Muñoz, D.; de Graaf, C.; Illas, F. Putting Error Bars on the Ab Initio Theoretical Estimates of the Magnetic Coupling Constants: The Parent Compounds of Superconducting Cuprates as a Case Study. J. Comput. Chem. 2004, 25, 1234-1241

49 Frisch, M. J.; Trucks, G. W; et al. Gaussian 09, Revision A.1; J. Gaussian, Inc.: Wallingford CT, 2009. 


\section{Graphic for TOC}

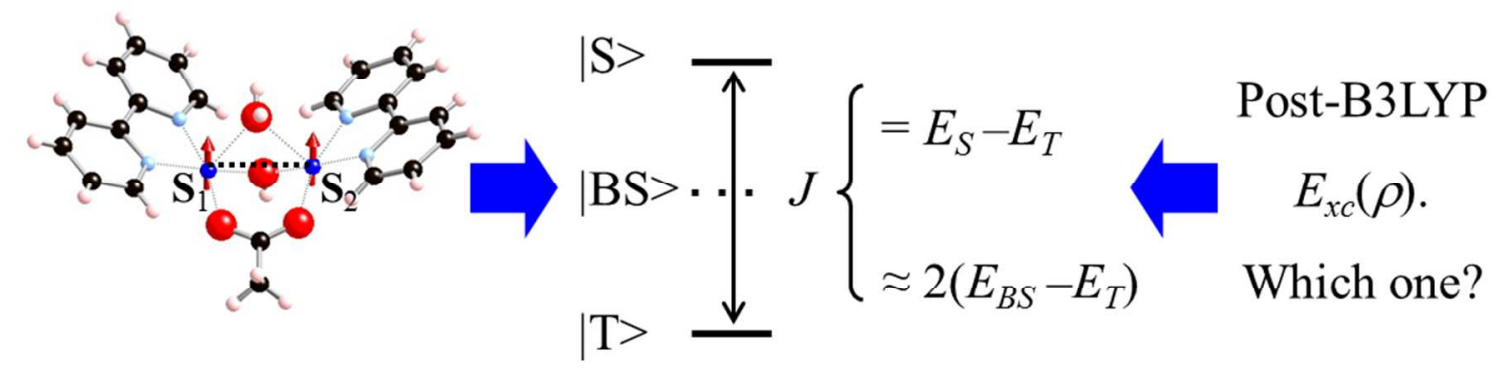

The reliability of calculated $J$ values using post-B3LYP functionals is system and range dependent. 